\title{
CDISC SDTM Left Ventricular Ejection Fraction Measurement Result Terminology
}

National Cancer Institute

\section{Source}

National Cancer Institute. CDISC SDTM Left Ventricular Ejection Fraction Measurement Result Terminology. NCI Thesaurus. Code C101868.

Terminology associated with the left ventricular ejection fraction measurement result codelist of the Clinical Data Interchange Standards Consortium (CDISC) Study Data Tabulation Model (SDT M). 